\title{
ESTUDIOS
}

\section{El nuevo Estatuto de Autonomía de Andalucía}

\section{Antonio Porras Nadales}

Palabras clave: Autonomía, Estado autonómico, Estatuto, Derechos sociales, Instituciones, Competencias, Financiación, Reforma.

Key words: Autonomy, Autonomic State, Estatute, Social rights, Institutions, Competences, Financing, Reform.

\section{Introducción}

La ratificación por referéndum el 18 de febrero de 2007 del nuevo Estatuto de Autonomía parece marcar una nueva etapa en la historia política de la Comunidad andaluza. Con 250 artículos divididos en diez Títulos más once disposiciones adicionales, el nuevo Estatuto constituye un texto normativo extenso que en algunos aspectos recuerda más bien a una auténtica Constitución. Sin embargo la amplitud de sus contenidos normativos no se traduce en una renovación sustancial del sistema institucional vigente, pues aparte de la incorporación de elementos que ya estaban presentes en leyes anteriores y de la relativa abundancia de reiteraciones, sus principales líneas de innovación se limitan en general a recoger la pauta marcada por el anterior Estatuto de Cataluña (y continuada por el resto de modificaciones estatutarias de otras Comunidades), al menos en sus aspectos más sustanciales como la introducción de una carta de derechos sociales (Título I), la enumeración expansiva del sistema de competencias (Título II) o la previsión de todo un sistema de relaciones institucionales de la Comunidad (Título IX). En el resto destaca fundamentalmente un intento de actualización general mediante la introducción de toda una serie de desarrollos legales que habían tenido lugar con anterioridad y que ahora se incorporan a la normativa fundamental, así como un esfuerzo de renovación de los discursos en lo que podríamos calificar como una tarea de dimensión más bien semántica o retórica.

En general, el criterio que siguen las principales innovaciones parece ajustarse a una pauta "estatocéntrica": es decir, procediendo a una incorporación al Estatuto de determinados elementos normativos o institucionales que ya existían con anterioridad en la propia Constitución española de 1978. Dicho en sentido negativo, ello significa que no hay en rigor un esfuerzo de especialización o de innovación institucional diferenciada en la escala territorial, y en conse- 
cuencia daría la impresión de que las Comunidades Autónomas se siguen considerando a modo de "mini-estados" que reproducen de forma mimética el modelo estatal vigente regulado en la Constitución de 1978. Así sucede por ejemplo con las leyes de mayoría absoluta, equivalentes a las leyes orgánicas de la Constitución (artículo 108 del Estatuto), o con la incorporación de la figura de los decretos legislativos y decretos-leyes (artículos 109 y 110), así como con la aparición de un Consejo de Justicia de Andalucía (artículo 144) equivalente en la escala territorial al Consejo General del Poder Judicial.

Si este proceso mimético refleja en primer lugar un escaso grado de creatividad o de capacidad de innovación, el riesgo alternativo radicaría en la ausencia de mecanismos al servicio de una adecuada especialización funcional entre las escalas central y autonómica: lo que al cabo de más de un cuarto de siglo de desarrollo autonómico constituye probablemente una demanda congruente a la vista del distinto tipo de tareas que -se supone- deben enfrentar las esferas central y autonómica en el sistema territorial español.

El texto aparece precedido de un difuso Preámbulo donde indirectamente se hace referencia a la que ha sido una de las principales causas de debate durante su proceso de elaboración, la consideración de Andalucía como una "realidad nacional", ponderando (igual que hace el Estatuto catalán) sus elementos de identificación colectiva a lo largo de la historia; se justifica, en segundo lugar, la necesidad de reformas en el intento de profundizar el autogobierno, aludiéndose también de forma contradictoria al perfeccionamiento de los mecanismos de cohesión territorial, solidaridad y cooperación institucional (mecanismos que evidentemente no dependen de una norma estatutaria al proyectarse sobre el conjunto del territorio del estado).

\section{Los objetivos programáticos}

En cualquier caso el perfil predominante del tipo de tareas $u$ objetivos programáticos que deberán atenderse desde la esfera institucional autonómica se sigue ajustando, según el nuevo Estatuto, al modelo propio del estado de bienestar, siguiendo en general la línea de evolución que caracteriza al estado social en Europa; lo que en este caso resulta particularmente reforzado a partir de la declaración de derechos sociales, donde se expande considerablemente el tipo de valores u objetivos finalistas a los que deberá atender la Junta de Andalucía.

Sin embargo debe partirse de la constatación de que el horizonte programático de la Comunidad, recogido en principio en el artículo 10 del nuevo Estatuto (dentro del Título Preliminar) que define los objetivos básicos de la misma, pese a experimentar en principio un considerable ensanchamiento al integrar hasta veinticuatro apartados (que serían una especie de réplica histórica a los once objetivos finalistas del anterior artículo 12. 3 del Estatuto de 1981), refleja al final una sustancial continuidad al cabo del tiempo. Si a comienzos de la década de los ochenta la categoría mágica en Andalucía parecía ser la noción del desarrollo económico (nacida obviamente como respuesta al diagnóstico previo del subdesarrollo) el perfil programático dominante un cuarto de siglo después parece situarse en torno a las nociones de calidad y de cohesión social. Sin embargo el cambio en el estilo, la introducción de nuevos matices o la invocación a categorías novedosas como la innovación, la sociedad del conocimiento, la participación o 
la capacidad emprendedora, no consiguen ocultar una sustancial continuidad del bloque programático, como se demuestra en la reiteración (con fórmulas ligeramente rectificadas) de las principales categorías finalistas que estaban ya presentes en el anterior Estatuto: pleno empleo, educación, desarrollo endógeno, retorno de emigrantes, calidad de vida, cohesión territorial, sistema de comunicaciones, respeto del paisaje (o del medio ambiente), desarrollo industrial y desarrollo rural. Mientras que la radical invocación a la superación de los desequilibrios territoriales del estado recogida en el anterior 12. 3. $9^{\circ}$, es sustituida ahora por la más difusa referencia a la convergencia con Europa del artículo 10. 3. $9^{\circ}$. Puede comprobarse pues que, en rigor, los horizontes finalistas o programáticos de Andalucía no han experimentado al cabo de un cuarto de siglo una transformación tan sustancial como en principio podría pensarse y que en consecuencia los cambios se sitúan más bien en torno a novedades de estilo o determinadas actualizaciones de carácter complementario.

En su desagregación sistemática se comprueba en primer lugar que el artículo 10.3 mantiene el horizonte originario del desarrollo económico que se proyecta en torno al pleno empleo $\left(1^{\circ}\right)$, desarrollo sostenible y solidario $\left(5^{\circ}\right)$, mejora de la calidad de vida $\left(7^{\circ}\right)$, convergencia $\left(9^{\circ}\right)$, desarrollo innovador y de calidad $\left(11^{\circ}\right)$ y desarrollo rural $\left(13^{\circ}\right)$. En segundo lugar aparecería el objetivo de la vertebración o cohesión social tanto en su proyección socioterritorial $\left(8^{\circ}\right)$, comunicaciones $\left(10^{\circ}\right)$, superación de la exclusión social $\left(14^{\circ}\right)$, integración de inmigrantes $\left(17^{\circ}\right)$, presencia participativa del tejido social $\left(19^{\circ}\right)$, concertación social $\left(20^{\circ}\right)$ e integración de minorías $\left(21^{\circ}\right)$. En tercer lugar aparecería el bloque finalista de tipo asistencial referido a situaciones de dependencia $\left(15^{\circ}\right)$ y discapacitados $\left(16^{\circ}\right)$, abarcando también la vuelta de los emigrados $\left(6^{\circ}\right.$ ). Y finalmente el horizonte de tipo modernizador que incide en los ámbitos inmateriales de la educación $\left(3^{\circ}\right)$, modalidad lingüística $\left(4^{\circ}\right)$, proyección del pluralismo en los medios de comunicación $\left(18^{\circ}\right)$, fomento de la cultura de la paz $\left(22^{\circ}\right)$, cooperación internacional $\left(23^{\circ}\right)$ y conocimiento de la historia andaluza $\left(24^{\circ}\right)$, hasta culminar con la incorporación a la sociedad del conocimiento $\left(12^{\circ}\right)$.

Por otra parte debe recordarse que este horizonte finalista experimenta una curiosa reiteración en el extenso artículo 37 (integrado dentro del Título I, referido a los derechos sociales y políticas públicas) donde se recoge una amplia serie de principios rectores de las políticas públicas, que en principio se supone deben servir tanto para el logro de los objetivos básicos de la Comunidad recogidos en el artículo 10.3 como para asegurar el pleno ejercicio de los derechos sociales: se trata de un total de veinticinco principios rectores de las políticas públicas, donde más que la dimensión instrumental vuelve a predominar nuevamente la estrictamente programática. Lo que refleja un cierto déficit de sistemática en el diseño del sistema general de fines, derechos y principios.

En este artículo 37 aparecen en primer lugar una serie de principios que se sitúan en torno a un perfil asistencial en relación con la atención a los mayores $\left(3^{\circ}\right)$, dependientes $\left(4^{\circ}\right)$, discapacitados $\left(5^{\circ}\right)$, sordos $\left(6^{\circ}\right)$, marginados $\left(7^{\circ}\right)$, jóvenes $\left(8^{\circ}\right)$, inmigrantes $\left(9^{\circ}\right)$, víctimas de delitos $\left(24^{\circ}\right)$, así como a situaciones de emergencia o catástrofe $\left(25^{\circ}\right)$. El segundo bloque sería al referido al desarrollo económico sostenible, abarcando investigación, desarrollo e innovación $\left(13^{\circ}\right)$, prioridad de los sectores turístico y agroalimentario $\left(14^{\circ}\right)$, acceso a la sociedad del conocimiento $\left(15^{\circ}\right)$, consumo responsable y solidario $\left(19^{\circ}\right)$, impulso a energías renovables $\left(21^{\circ}\right)$, respeto al medio ambiente $\left(20^{\circ}\right)$ y uso racional del suelo $\left(22^{\circ}\right)$. Un tercer bloque sería el orientado hacia el marco de la 
vertebración social integrando las relaciones laborales, que afectarían al empleo de calidad $\left(10^{\circ}\right)$, equiparación laboral $\left(11^{\circ}\right)$, concertación social $\left(12^{\circ}\right)$, y fomento del asociacionismo social $\left(16^{\circ}\right.$ ). Finalmente los elementos inmateriales o culturales afectarían tanto a la lucha contra el sexismo y la xenofobia $\left(2^{\circ}\right)$, libre acceso a la cultura $\left(17^{\circ}\right)$, conservación del patrimonio cultural $\left(18^{\circ}\right)$ y respeto a la diversidad cultural $\left(23^{\circ}\right)$. Por último, cabría sugerir que en rigor el único elemento auténticamente instrumental sería el que se recoge en el epígrafe $1^{\circ}$, la prestación de unos servicios públicos de calidad, que en todo caso tiene una clara interconexión con el derecho a la buena administración del artículo 31.

\section{La "parte dogmática": los derechos sociales}

La introducción de una carta de derechos sociales que se concretan fundamentalmente en el Capítulo II del Título II constituye una primera novedad estatutaria, aunque claramente influida por el anterior texto catalán, integrando un total de veintidós artículos (del 15 al 36) de desigual extensión. Por supuesto debe considerarse que se trata de una novedad relativa pues gran parte de tales derechos existen ya en normas de superior rango (la Constitución o la carta de derechos de Niza) o bien han sido objeto de desarrollos legales previos.

3.1. Una ordenación clasificatoria elemental nos permitiría en primer lugar aludir al bloque de derechos de dimensión personal que afectan a la regulación de nuevos modelos de convivencia social a partir de la configuración de espacios de libertad individual: igualdad de género (artículo 15), regulación del testamento vital y derecho a una muerte digna (artículo 20), protección de datos (artículo 32), derecho de acceso a las nuevas tecnologías (artículo 34) y a la libre orientación sexual (artículo 35). En teoría constituyen típicos derechos de libertad personal que no requerirían de políticas intervencionistas orientadas a asegurar su garantía; sin embargo la predominancia del perfil asistencial o prestacional se detecta en este ámbito con previsiones de acción pública en sectores como la orientación sexual o la responsabilidad de las instituciones sanitarias en los tratamientos del dolor y cuidados paliativos integrales ante el proceso de la muerte. Igualmente se prevén medidas preventivas, asistenciales y ayudas públicas en relación con el derecho de las mujeres a una protección integral contra la violencia de género del artículo 16, así como en relación con el artículo 17 referido a la protección de la familia (aunque en rigor este último parece contemplar más bien la regulación de nuevas modalidades de familia así como el status de las parejas no casadas inscritas en el correspondiente registro público). Estaríamos pues ante unos derechos de "última generación" que se proyectan en una clara dimensión de libertad personal, conformando bien nuevos modelos de ordenación de la convivencia social, - bien nuevas formas de enfrentarse a algunos de los riesgos de la vida moderna (como los supuestos de la protección de datos o el derecho de acceso a las nuevas tecnologías).

3.2. Un segundo bloque de derechos sería el que configura hasta el presente el perfil clásico de las principales políticas intervencionistas de la Junta de Andalucía integrando las materias de educación, salud, vivienda, trabajo, medio ambiente y cultura; ámbitos donde en gran medida la tarea del nuevo Estatuto parece consistir en incorporar derechos que han sido ya objeto de 
desarrollo legal con anterioridad. Acaso los principales, en términos de actividad prestadora de los poderes públicos, serían los que se recogen en los artículos 21 y 22, educación y salud, que responden a estilos y sistemáticas muy diferentes: mientras el artículo 22 referido al derecho a la salud contiene una enumeración sistemática de derechos declarados acompañado de la correspondiente previsión de mediación legal en su apartado 3 ("Con arreglo a la ley se establecerán los términos, condiciones y requisitos del ejercicio de los derechos previstos en los apartados anteriores"); en cambio, el artículo 21 referido al derecho a la educación contiene una redacción algo más extensa y tortuosa (acaso reflejo de su propio proceso de elaboración) donde se mezclan confusamente categorías de derechos declarados con la reiterada expresión enfática "se garantiza" y previsiones de mera política educativa.

Junto a los derechos a la educación y a la salud, que conforman en la práctica el ámbito más sustancial de la actividad intervencionista de la Junta de Andalucía, resulta sorprendente la escasa relevancia atribuida al derecho a la vivienda, pues el artículo 25 se limita lacónicamente a afirmar: "Para favorecer el ejercicio del derecho constitucional a una vivienda digna y adecuada, los poderes públicos están obligados a la promoción pública de la vivienda. La ley regulará el acceso a la misma en condiciones de igualdad, así como las ayudas que lo faciliten." Parece pues que el estatuyente, tan generoso en su reconocimiento de valores sociales relacionados con la calidad de vida y la atención a colectivos desfavorecidos, tiene algunas precauciones a la hora de enfrentarse a una política que hasta ahora sólo ha sido capaz de demostrar reiteradas insuficiencias (cuando no de suscitar numerosos casos de corrupción).

En cambio, la materia medioambiental tiene una regulación más amplia y sistemática ya que junto al artículo 28 que recoge los derechos en materia de medio ambiente, se integran otra serie de previsiones normativas en distintas partes del nuevo Estatuto. Este artículo 28 se refiere al derecho de todas las personas a vivir en un medio ambiente equilibrado, sostenible y saludable, y a disfrutar de los recursos naturales, del entorno y el paisaje, imponiendo al mismo tiempo el deber de un uso responsable del mismo. Junto al derecho a la información medioambiental se prevé como instrumento de garantía la protección de la diversidad biológica y los procesos ecológicos, el patrimonio natural, el paisaje, el agua, el aire y los recursos naturales. La materia de protección medioambiental constituye igualmente no sólo uno de los instrumentos previstos para la consecución del objetivo básico de la mejora de la calidad de vida de los andaluces (artículo 10), implicando la gestión del agua, también sino uno de los principios rectores de las políticas públicas (según el apartado $20^{\circ}$ del artículo 37,1 ), así como un deber de las empresas que desarrollen su actividad en Andalucía, las cuales deberán ajustarse a los principios de respeto y conservación del medio ambiente, según el artículo 36.2. Pero sobre todo, debe recordarse que el medio ambiente es objeto de un Título específico, el VII (artículos 195 a 206), en lo que constituye probablemente la única política pública regulada como tal en el texto del nuevo Estatuto de Autonomía.

El derecho de acceso a la cultura del artículo 33 presenta una regulación relativamente lacónica que viene a confirmar su perfil más propio de una auténtica política pública, que se complementa con la previsión del deber de cuidar y proteger el patrimonio público de carácter histórico-artístico (artículo 36, 1, f). Se constata así la escasa proyección subjetiva de este derecho frente a su dimensión predominantemente programática. 
En cuanto al paquete de derechos de proyección socioeconómica integra el derecho al trabajo (artículo 26, con cuatro previsiones referidas al acceso gratuito a los servicios públicos de empleo, acceso al empleo público en condiciones de igualdad y según los principios constitucionales de mérito y capacidad, acceso a la formación profesional y derecho al descanso y al ocio), junto con una previsión acerca de la presencia participativa de las organizaciones sindicales y empresariales más representativas en el ámbito de la Junta de Andalucía. Debe recordarse que la materia sociolaboral se incluye también en un capítulo específico (el segundo del Título VI dedicado a economía, empleo y hacienda, artículos 166 a 174). Igualmente se reconoce con bastante laconismo el derecho de los consumidores (artículo 27) que integra los derechos a asociarse, a la información, formación y protección, con previsión de un catálogo de derechos del consumidor que se desarrollará por vía legal.

3.3. Un siguiente grupo de derechos sería el que integra el perfil estrictamente asistencial, más directamente orientado hacia colectivos desfavorecidos y donde lógicamente la dimensión declarativa se funde más intensamente con la prestacional. Así aparecen las referencias a menores y mayores (artículos 18 y 19) a quienes se les reconoce el derecho a recibir de los poderes públicos una protección y una atención integral tanto para su bienestar en el ámbito familiar, escolar y social en el caso de los menores, como para asegurar una vida digna e independiente en el caso de los mayores, incluyendo el derecho de acceso a una atención gerontológica adecuada. En ambos supuestos con previsión de percibir las prestaciones que establezcan las leyes. El artículo 24, referido a las personas con discapacidad, incluye el derecho de acceder a las ayudas, prestaciones y servicios necesarios para su desarrollo personal y social. Finalmente el artículo 23 contempla el derecho general a acceder a las prestaciones de un sistema público de servicios sociales que ahora se reconoce con un carácter universal o "derecho de todos", integrando la previsión en su apartado segundo de una renta básica que garantice unas condiciones de vida digna. Se constata así la prioridad estatutaria del derecho genérico a la asistencia social implicando un sistema público de servicios, con una remisión a la ley de la posterior regulación específica del derecho a una renta básica o "salario social".

3.4. Cabría hacer referencia finalmente a dos novedades de esa carta de derechos: por una parte los derechos de proyección política y por otra el apartado relativo a los deberes. Dentro de la categoría de los derechos políticos aparece en primer lugar el derecho de participación recogido en el artículo 30 que remite en parte a la redacción del artículo 23 de la Constitución, incluyendo el derecho de sufragio activo y pasivo, el de iniciativa legislativa y participación en la elaboración de las leyes (como remisión en este caso al Reglamento del Parlamento), el de promover la convocatoria de consultas populares, el derecho de petición y finalmente el derecho a participar en la vida pública. En alguna medida se complementaría este apartado con el anterior artículo 29, referido al derecho de acceso a la justicia gratuita. Una singular novedad representa en este campo el derecho a una buena administración recogido en el artículo 31, con un contenido de mayor originalidad y amplitud donde se integran por una parte la exigencia de proporcionalidad en la actuación de las administraciones públicas así como el derecho de todos a participar plenamente en las decisiones que les afecten, a obtener una información veraz, y a que sus asuntos se traten de manera objetiva e imparcial y sean resueltos en un plazo razonable, incluyendo igualmente el derecho a acceder a los archivos y registros de las instituciones, corporaciones, órganos y organismos públicos de Andalucía. 
En cuanto al apartado de los deberes se agrupan inicialmente en el artículo 36 donde, sobre la base de un desarrollo legal previo, se prevé la obligación de todos a contribuir al sostenimiento del gasto público en función de sus ingresos, a conservar el medio ambiente, a colaborar en las situaciones de emergencia, a cumplir las obligaciones derivadas de la participación de los ciudadanos en la Administración electoral, a hacer un uso responsable y solidario de las prestaciones y servicios públicos colaborando en su buen funcionamiento, a cuidar y proteger el patrimonio público especialmente el de carácter histórico-artístico y natural, y a contribuir a la educación de los hijos, especialmente en la enseñanza obligatoria. Pero junto a las previsiones explícitas del artículo 36 hay igualmente otros deberes que se recogen de forma dispersa en el articulado como el deber de hacer un uso responsable del medio ambiente para evitar su deterioro y conservarlo para las generaciones futuras del artículo 28, 1, o el de respetar y preservar el patrimonio cultural andaluz del artículo 33.

\section{Las garantías de los derechos}

El Capítulo IV de este Título I se dedica a regular el sistema de garantías de los derechos sociales siguiendo un diseño que en general se aproxima con claridad al modelo de la Constitución de 1978.

En primer lugar debe atenderse a la innovación contenida en el artículo 38 referida a la fuerza vinculante horizontal que tienen los derechos declarados en el artículo 14 y Capítulo II, es decir, frente a los particulares; aunque ello dependiendo naturalmente "de la naturaleza de cada derecho"; una precisión particularmente evidente si consideramos que la mayoría de ellos son derechos de bienestar o de prestación cuya responsabilidad activa se proyecta sobre la esfera pública.

A partir de aquí el sistema de garantías recoge las dos principales vías contenidas en el artículo 53 de la Constitución: (a) la vinculación del legislador autonómico al contenido de los derechos declarados en el nuevo Estatuto y (b) la vía jurisdiccional ordinaria según la legislación estatal (sin que en principio se establezcan parámetros de preferencia o sumariedad similares al amparo). Con independencia de que la reproducción del modelo constitucional no sea completa, debe en todo caso ponderarse positivamente tanto el establecimiento de un parámetro de control al legislador autonómico como la generalización de un sistema judicial de control.

Más allá de este diseño sólo cabría recordar que en rigor los instrumentos judiciales mantienen normalmente su plena eficacia en relación con los tradicionales derechos de "libertad" (o "libertad negativa" para ser más precisos) implicando el establecimiento de límites a los poderes públicos. Sin embargo su utilidad final será siempre relativa cuando nos enfrentamos a derechos de prestación que exigen una actividad intervencionista de los poderes públicos. De donde cabe destacar finalmente la centralidad operativa que en este trascendental bloque del nuevo Estatuto acabará teniendo la figura del Defensor del Pueblo debido a su mayor capacidad de incidencia positiva sobre la esfera intervencionista de las administraciones públicas. 
Si en su configuración general el sistema de garantías puede considerarse pues como suficiente, siguiendo la pauta de adecuación al modelo constitucional, debe recordarse en todo caso que el nuevo Estatuto de Autonomía para Andalucía tampoco ha logrado conseguir, como era previsible, la cuadratura del círculo: es decir, el establecimiento de un sistema de control por omisión que tutele la "no-acción" de los poderes públicos, ya sean el legislativo o el ejecutivo y la administración. La observación tiene en este caso una peculiar relevancia si recordamos nuevamente que la mayor parte de los derechos reconocidos en el nuevo Estatuto no son derechos individuales de libertad (imponiendo una limitación a los poderes públicos) sino justo lo contrario, es decir, derechos de prestación que requieren como vía esencial para adquirir eficacia el desarrollo de políticas públicas eficaces.

En cualquier caso una valoración final del sistema de garantías nos confirmaría el considerable avance introducido a partir de la vinculación del legislador y la vía jurisdiccional de tutela de los derechos reconocidos en el Capítulo II del Título l. En cambio, no parecen haberse introducido previsiones explícitas referidas a las modificaciones necesarias en los "otros" instrumentos de garantía, los que afectan a la propia acción intervencionista, es decir, en el ámbito de la administración y el sector público de los que dependerá en última instancia el desarrollo de las prestaciones y servicios públicos necesarios para dar cobertura a tan amplio marco declarativo. Sólo el proceso de desarrollo y aplicación efectiva del Estatuto nos demostrará en su caso la operatividad final de tales instrumentos, contando con el decisivo protagonismo activo del Defensor del Pueblo Andaluz.

\section{El sistema institucional}

Para atender a este amplio panorama de fines y valores se supone en cualquier caso que la Comunidad Autónoma de Andalucía deberá desplegar todo un complejo instrumental que incluye en primer lugar el sistema competencial, en segundo lugar el propio sistema institucional y burocrático, y finalmente los recursos financieros que integran la hacienda de la Comunidad Autónoma.

Comenzando con el sistema institucional, regulado en el Título IV, cabría afirmar que la principal novedad consiste en la ausencia de novedades. Por supuesto debe partirse de la constatación de que los sistemas autonómicos españoles han optado hasta ahora por la generalización del modelo parlamentario vigente en la Constitución española, que parece pues consolidarse históricamente. En el caso de Andalucía debería comenzarse recordando las insuficiencias del primer Estatuto ${ }^{1}$ que han obligado a lo largo del tiempo a un desarrollo parcial del sistema de gobierno parlamentario -o de algunos de sus instrumentos, como la disolución- por vía legal. En todo caso y pese a la introducción de la potestad de disolución por vía legal, hay que recordar que el procedimiento de investidura seguía careciendo hasta ahora de un mecanismo de cierre capaz de impedir un gobierno en minoría, como el que paralizó institucionalmente a Andalucía durante el llamado periodo de la "pinza", de 1994 a 1996.

\footnotetext{
' A. Porras, J. J. Ruiz-Rico (eds.) (1990), El Estatuto de Andalucía. Estudio sistemático, Barcelona, Ariel.
} 
Por lo tanto la normalización del sistema parlamentario introducida por el nuevo Estatuto parece implicar la definitiva consolidación del modelo establecido en la Constitución de 1978, con un ejecutivo protegido por los instrumentos de estabilidad que se diseñaron en el parlamentarismo racionalizado durante las primeras décadas del siglo XX hasta culminar en el modelo alemán. Este poder ejecutivo surgirá ahora de un proceso de investidura configurado de forma completa, con su correspondiente cláusula de disolución automática; aparece dotado de una clara centralidad presidencial y estará sometido a los instrumentos clásicos de control parlamentario tal y como se han venido configurando al cabo de casi un cuarto de siglo de vigencia de nuestro sistema autonómico.

La otra cara de este proceso de normalización parlamentaria afectaría alternativamente a la ausencia de innovaciones sustanciales relativas al diseño del sistema institucional y de la forma de gobierno ${ }^{2}$ : desde esta otra perspectiva cabe recordar que en el nuevo texto ni se establecen limitaciones a los mandatos presidenciales, ni se prevén instrumentos específicos para hacer efectiva una eventual responsabilidad autónoma de los consejeros individualmente considerados (permitiendo en su caso la correspondiente reprobación), ni hay modificaciones sustanciales en la propia configuración estructural del gobierno o en sus relaciones con la administración.

El Parlamento de Andalucía se sigue considerando como un órgano central del sistema, con la principal novedad de que al no cerrarse el número de sus componentes en el Estatuto se abre la vía para permitir en su caso una mejor presencia de ciertas minorías. El sistema electoral no presenta grandes novedades, a salvo posibles innovaciones complementarias que se introduzcan por vía legal, manteniéndose las pautas de la circunscripción provincial y el sistema proporcional.

Una novedad relativa sería la introducción de las consultas populares, según los artículos 78 y 111.3 , categoría tras la que se oculta de forma más o menos elusiva el instrumento del referén$d \mathrm{~m}^{3}$. Estamos evidentemente ante un desbordamiento semántico que abre las puertas a fórmulas más o menos experimentales de democracia participativa que podrán operar tanto en la escala local como en la estrictamente autonómica. Aunque ciertamente la esfera local, a la que en principio se dedica el Título III, no parece que vaya a adquirir un protagonismo notable en un futuro inmediato pues la regulación del nuevo Estatuto mantiene las pautas anteriores y comparadas de reforzamiento de la centralidad autonómica frente al ámbito local o comarcal.

En cuanto a la figura del Presidente de la Junta de Andalucía, destaca en principio la relativa ausencia de innovaciones, si exceptuamos la normalización de su procedimiento de investidura con la introducción del mecanismo de la disolución automática. Sus competencias se siguen ajustando a las pautas ya conocidas: dirección y coordinación de la actividad del gobierno, nombramiento y cese de sus miembros, coordinación de la administración y suprema representación de la Comunidad y del Estado en Andalucía. El sistema de relaciones entre el Parlamento

\footnotetext{
2 Sobre el tema cfr. G. RuIz-Rico (coord.) (2003), El Presidente de la Junta de Andalucía y el Consejo de Gobierno, Parlamento de Andalucía, Comares, Granada.
}

${ }^{3}$ Sobre el tema N. PÉREZ Sola (1994), La regulación constitucional del referéndum, Universidad de Jaén. 
y el ejecutivo no presenta grandes novedades salvo la incorporación normalizada de la potestad presidencial de disolución, siguiendo igualmente las pautas de la propia Constitución.

Una innovación puramente formal sería la introducción de toda una serie de instituciones surgidas de desarrollos legales previos, que en algunos casos han adquirido ya un notable grado de consolidación institucional (Defensor del Pueblo, Cámara de Cuentas, Consejo Consultivo, Consejo Económico y Social y Consejo Audiovisual); se trata de instituciones de control que se vienen integrando normalizadamente en el organigrama de la Junta de Andalucía desde hace tiempo, acaso con la única excepción del más reciente Consejo Audiovisual. Tampoco parece dar la impresión de que el nuevo texto estatutario refleje una apuesta consistente por el reforzamiento de instituciones o agencias independientes, pues llama la atención no sólo el reiterado desplazamiento a las correspondientes normas legales de sus sistemas de nombramiento (con la excepción del Defensor del Pueblo) sino también la escasa insistencia en los principios de autonomía e independencia que deberían presidir su configuración.

En cuanto al Capítulo VII y final de este Título IV, dedicado a la Administración de la Junta de Andalucía, constituye igualmente una novedad formal que trata de reflejar el alto grado de desarrollo alcanzado por la administración autonómica, de la que prácticamente apenas se contenían previsiones normativas en el anterior Estatuto. Su redacción parece reflejar un precario equilibrio entre tendencias inerciales o conservadoras orientadas a mantener un sistema burocrático de corte más o menos tradicional, frente a tendencias innovadoras o modernizadoras que tienen un desarrollo bastante puntual. El mejor reflejo de tal equilibrio probablemente se encuentre en el artículo 133.1 que recoge los principios de actuación de la administración mediante una larga serie enumerativa donde se integran los principios de: eficacia, eficiencia, racionalidad organizativa, jerarquía, simplificación de procedimientos, desconcentración, coordinación, cooperación, imparcialidad, transparencia, lealtad institucional, buena fe, protección de la confianza legítima, no discriminación y proximidad a los ciudadanos. Una serie enumerativa cuya extensión y heterogeneidad parece reflejar una cierta carencia de sistemática y un criterio ecléctico o meramente agregativo, de escasa operatividad práctica.

Como elementos innovadores aparecen en todo caso la reserva de género en el nombramiento de órganos directivos, la referencia a las cartas de servicios del artículo 137 y la previsión de una ley reguladora del sistema de evaluación de políticas públicas (artículo 138). Obsérvese que es éste, el artículo 138, el único que hace referencia en este Capítulo a la noción de políticas públicas, mientras que en el artículo de cabecera (el 133) se habla tan sólo de servicios, prestación de servicios o gestión de competencias, reflejando con claridad el confuso equilibrio entre dos distintas filosofías a la hora de regular la administración de la Junta de Andalucía ${ }^{4}$.

Finalmente el bloque dedicado a la administración de justicia se recoge en el Título $\mathrm{V}$ del nuevo Estatuto, con una dimensión regulativa más extensa debido seguramente a que se trata de uno de los ámbitos de mayor tensión competencial; lo que da lugar a la peculiaridad de que el Capítulo III de este Título se dedique a ampliar específicamente las previsiones del artículo 80, donde se definen las competencias compartidas en materia de justicia, situadas fundamentalmente en torno a un triple ámbito: recursos materiales, recursos humanos y delimitación territorial interna.

\footnotetext{
${ }^{4}$ Sobre el tema cfr. A. PORRAS (2005), "La reforma de la administración andaluza y los desafíos de la Segunda Modernización", Revista de Fomento Social, n 237.
} 
Este desarrollo competencial viene precedido por dos capítulos de contenido orgánico referidos respectivamente al Tribunal Superior de Justicia de Andalucía (Capítulo I) y al Consejo de Justicia de Andalucía (Capítulo II) donde aparecen algunas sustanciales novedades. En primer lugar la consideración del Tribunal Superior de Justicia de Andalucía como última instancia jurisdiccional de todos los procesos judiciales iniciados en Andalucía, reservando la labor del Tribunal Supremo a la mera unificación de doctrina en los términos que establezca la Ley Orgánica del Poder Judicial. Se trata ciertamente de una modificación general del sistema que sólo puede tener consistencia mediante su apoyo en la citada Ley Orgánica, donde se expresa la estrategia de reordenación territorial del sistema de justicia en España.

Además de reiterar las competencias específicas del TSJA en el artículo 142, el nuevo texto estatuario entra en una regulación más detallada (artículo 143) no sólo del nombramiento de su Presidente sino también de los Presidentes de Sala y del Fiscal Superior. Mayor complejidad, por sus dudas de potencial inconstitucionalidad, reviste la creación del Consejo de Justicia de Andalucía, concebido en principio como órgano desconcentrado del Consejo General del Poder Judicial y cuyos miembros serán elegidos entre jueces, magistrados, fiscales y juristas de reconocido prestigio según las previsiones de la Ley Orgánica del Poder Judicial. La enumeración de sus atribuciones en el ámbito jurisdiccional andaluz se situarían en la órbita de las actividades inspectivas y disciplinarias, así como de informe y propuesta, con una ubicación institucional algo confusa entre el Consejo General del Poder Judicial, el Consejo de Gobierno y el propio Parlamento de Andalucía al que debe presentar una memoria anual.

En cuanto al Capítulo III referido a las competencias de la Junta en materia de administración de justicia, centradas fundamentalmente en medios personales, materiales y demarcación territorial, entra en una enumeración acaso excesivamente detallada de estos aspectos, con previsiones adicionales referidas a justicia gratuita, justicia de pazy de proximidad, participación ciudadana y carta de derechos ciudadanos.

\section{Competencias y financiación}

El sistema competencial se encuentra regulado en el amplio Título II, artículos 42 a 88 , redactado siguiendo la pauta catalana, es decir, mediante una desagregación de detalle en cada materia, con el objetivo -se supone- de evitar ambigüedades interpretativas que permitan la interferencia del estado central. En cualquier caso se contempla ahora un criterio clasificatorio donde se distingue entre competencias exclusivas, compartidas, ejecutivas, así como competencias en relación con la aplicación del derecho comunitario y competencias que aunque no contempladas expresamente en el Estatuto pueden ser transferidas o delegadas por el Estado.

Las competencias exclusivas, integrando potestad legislativa, reglamentaria y función ejecutiva, no tienen sin embargo carácter "excluyente, es decir, no excluyen la incidencia de ámbitos competenciales del estado derivados del artículo 149.1 de la Constitución. Las competencias compartidas tienen los mismos contenidos, legislativo, reglamentario y ejecutivo, pero en relación con los casos en que la Comunidad Autónoma debe ejercer sus competencias a partir de las bases que establezca el Estado en determinadas materias, igualmente según el artículo 149.1 
de la Constitución. La innovación en este punto reside en el intento de limitar el alcance de las bases garantizando la potestad de la Comunidad para establecer políticas propias, mediante una recepción de la "doctrina catalana" que defiende la noción de bases como meros "principios" (y por lo tanto no auténticas normas jurídicas) que operarían como directrices genéricas a desarrollar por la legislación autonómica en las distintas materias. Una visión doctrinal que no excluye sin embargo interpretaciones distintas, abriendo interrogantes en el desarrollo posterior del proceso autonómico. En cualquier caso será la enumeración concreta del texto la que determinará en cada supuesto (siempre en el marco de constitucionalidad) el carácter de cada ámbito material.

En cuanto a las competencias ejecutivas, incluyen la potestad de organización de la propia administración y las funciones y actividades que el ordenamiento atribuye a la Administración Pública así como la aprobación de disposiciones reglamentarias para la ejecución de la normativa del Estado. El carácter genérico de la definición implica pues cualquier tipo de reglamentos y no los meramente organizativos.

Las competencias en relación con la aplicación del derecho comunitario comprenden el desarrollo y la ejecución de la normativa de la Unión Europea cuando afecte al ámbito de las competencias de la Comunidad Autónoma; lo que implica la incorporación al Estatuto de Andalucía de la doctrina que viene aplicando el Tribunal Constitucional en relación con las competencias de las Comunidades Autónomas para desarrollar y ejecutar el derecho comunitario, según la cual tales facultades deben corresponder al ente que tenga la competencia para llevar a cabo la actuación de desarrollo normativo o ejecución de la que se trate sobre cada materia, conforme al sistema de reparto interno de competencias

Habría finalmente una posible categoría de competencias que puedan ser transferidas o delegadas por el Estado aunque no estén contempladas de forma expresa en el Estatuto (siguiendo igualmente la pauta catalana); así como competencias de participación en los procedimientos encaminados a la aprobación de normas, la adopción de decisiones de competencia del Estado o la designación de representantes en los organismos económicos y sociales del Estado, donde se incluirían tanto la participación en ámbitos de planificación económica o medidas de política general con incidencia en Andalucía como la designación de miembros de los órganos de dirección de ciertas instituciones (como Banco de España, Comisión Nacional del Mercado de Valores, Comisión del Mercado de las Telecomunicaciones y demás organismos que ejercen funciones de autoridad reguladora sobre materias de relevancia económica y social relacionadas con las competencias de la Comunidad Autónoma). Todo ello en el marco de la correspondiente legislación estatal sobre el tema.

En todo caso debe entenderse que la enumeración expansiva y detallada no supone en rigor una ampliación sustancial de las competencias disponibles (salvo posibles transferencias del estado) sino simplemente una clarificación en sentido expansivo de sus posibles contenidos dudosos para evitar interferencias estatales, quedando algunos aspectos (como las competencias sobre la confederación hidrográfica del Guadalquivir) pendientes de su posible y eventual inconstitucionalidad. 
El último de los circuitos instrumentales que se supone debe usar la Junta de Andalucía para alcanzar sus fines u objetivos programáticos se encuentra regulado en el Título VI, dedicado a economía, empleo y hacienda, donde se comprueba una de las más características manifestaciones del perfil tendencialmente "constitucional" del nuevo Estatuto, en el sentido de que contiene previsiones de regulación económica que en algunos casos parecen poner en entredicho el principio de unidad del mercado del estado español. Sus objetivos finalistas se definen globalmente en el artículo 157, aunque en general todo el capítulo I dedicado a economía destaca por su dimensión esencialmente programática, permitiendo en todo caso la creación de entes instrumentales o empresas públicas así como la ordenación del sistema financiero, con previsiones de participación en las tareas de planificación económica del estado.

El bloque dedicado a empleo y relaciones laborales del Capítulo II, igualmente con un contenido predominantemente declarativo, insiste también en la proyección del nuevo sistema de valores sobre el ámbito laboral, especialmente la igualdad de género (artículo 167) y la dimensión asistencial, que podrán afectar al sector de la contratación y la subvención pública.

Finalmente el amplio bloque dedicado a hacienda sigue también la pauta catalana de avanzar en un agotamiento normativo del tema, hasta el punto de que parecen limitarse las perspectivas y posibilidades de un sistema "general" de financiación territorial, con sus riesgos subsiguientes no sólo en clave de constitucionalidad sino en términos de operatividad del modelo territorial de financiación, atribuyéndosele un especial protagonismo a la comisión mixta de carácter bilateral sobre el tema (artículo 184). Como instrumento de gestión se prevé la creación de una Agencia Tributaria en el artículo 181 encargada de gestionar los tributos propios así como los estatales cedidos a la Junta, con posibilidades de colaborar en una gestión compartida con la agencia estatal.

La pretensión de regular exhaustivamente el sistema financiero en el Estatuto se complementa con otro de los aspectos que tuvieron un carácter más llamativo durante el debate, la regulación en la Disposición Adicional Segunda de la llamada "deuda histórica", que en todo caso implicará un acuerdo con el Estado.

\section{Las relaciones institucionales de la Comunidad Autónoma}

El Título IX del nuevo Estatuto se dedica a las relaciones institucionales de la Comunidad Autónoma mediante una amplia regulación que se refiere a las relaciones con el Estado, con otras Comunidades Autónomas, con las instituciones de la Unión Europea, con terceros países, así como a la cooperación al desarrollo, en lo que constituye una muestra de esa tendencia "estatomórfica" que caracteriza al nuevo modelo estatutario. En teoría se trataría de la innovadora hipótesis de posicionar activamente a Andalucía ante el conjunto de relaciones que configuran el inmediato futuro del sistema-red, particularmente ante el contexto europeo.

En el primer apartado, y a partir de la previsión del artículo 219,2 (según el cual "Para los asuntos de interés específico de la Comunidad Autónoma se establecerán los correspondientes instrumentos bilaterales de relación. En los asuntos de interés general, Andalucía participará a 
través de los procedimientos o en los órganos multilaterales que se constituyan"), la principal novedad parece residir en la amplia regulación que se prevé para la Comisión Bilateral en el artículo 220, a la que se atribuyen competencias con alta incidencia en la actividad general del gobierno del estado, como proyectos de ley, planificación económica general, designación de representantes en organismos del estado, o seguimiento de la política europea y de la acción exterior del Estado. Una previsión que, por más que se limite a repetir previsiones ya incluidas en el Estatuto catalán y aunque se declare inspirada en los principios de colaboración, cooperación, lealtad institucional y mutuo auxilio (artículo 219.1), suscita la emergencia de un difuso modelo confederal que implicaría una auténtica mutación del sistema constitucional vigente.

En cuanto a las relaciones con otras Comunidades Autónomas, se seguirán ajustando al sistema de convenios y acuerdos que requieren en cada caso la comunicación a las Cortes o su autorización previa, autorizándose en todo caso al ejecutivo autonómico para suscribir convenios de carácter cultural. El sistema de relaciones con la Unión Europea se proyecta tanto en la fase ascendente como a la descendente; nuevamente con previsión de participar de forma bilateral "en los asuntos que le afectan exclusivamente" en la formación de la voluntad del estado, incluso con un carácter "determinante" (artículo 231, 2 y 3), así como en las propias instituciones europeas, con presencia andaluza en las delegaciones españolas correspondientes. La fase descendente de desarrollo y ejecución del derecho europeo se asume igualmente por la Comunidad andaluza según lo previsto en el artículo 235.

La proyección de la Comunidad Autónoma en el ámbito de la acción exterior del estado implica no solamente un derecho de información sobre tratados internacionales sino igualmente la posibilidad de celebrar acuerdos de colaboración exterior así como la participación en organismos internacionales o en diversos foros con países fronterizos; un marco normativo que trata de normalizar la dinámica efectivamente desarrollada por nuestras Comunidades Autónomas en el ámbito exterior, aunque se ha echado de menos la ausencia de una proclamación específica de la proyección de Andalucía en el ámbito mediterráneo.

Finalmente las iniciativas de cooperación al desarrollo, inspiradas en el principio de solidaridad que proclama el artículo 245, deberán afectar especialmente a ámbitos más próximos como Iberoamérica, el Magreb y África, implicando una potestad de coordinar las acciones de corporaciones locales y otros entes públicos.

El panorama resultante parece confirmar una amplia presencia exterior de la Comunidad Autónoma: lo que seguramente suscita perspectivas positivas en un entorno de cooperación, o en supuestos de intercambios de tipo cultural, pero no deja de suscitar numerosos interrogantes cuando tales relaciones se sitúen en entornos potencialmente conflictivos (pensemos por ejemplo en las difíciles perspectivas a propósito del Sahara y Marruecos). 


\section{Aspectos complementarios}

Tan amplia panorámica normativa se complementa finalmente con tres Títulos originales, el VII dedicado a Medio Ambiente, el VIII dedicado a Medios de Comunicación Audiovisual, y el último y X, dedicado a la reforma del Estatuto (ya hemos hecho una breve referencia al III, referido a la organización territorial interna, carente de grandes novedades). En principio los Títulos VIl y VIII suscitan una cierta sorpresa por su carácter especializado que, en todo caso, en el primero de los supuestos puede entenderse como un intento de regulación estatutaria de los parámetros fundamentales de una política pública dotada de especial prioridad y complejidad; aunque de su texto se deduce fundamentalmente una fijación de ámbitos finalistas o programáticos más que una regulación de condiciones u objetivos concretos para la acción pública. Si esta filosofía justificaría la inclusión de un título sobre medio ambiente, la pregunta subsiguiente sería por qué no se incluyen otros de similar magnitud para algunas de las principales políticas de la Junta (como por ejemplo la educativa o la sanitaria): teóricamente se trataría tendencialmente de fijar una secuencia normativa de carácter sistemático que abarcaría tres bloques complementarios: (a) las normas programáticas y los derechos sociales o de dimensión prestacional (entendidos como horizonte finalista), (b) las competencias (entendidas como soportes habilitantes para la acción) y (c) las políticas públicas (entendidas como parámetros o coordenadas donde deberán operar los poderes públicos). Sin embargo tan ambiciosa pretensión sistemática (que cuenta en todo caso con el precedente del Tratado de la Constitución Europea, no ratificado) parece que sólo se contempla en relación con el ámbito medioambiental.

En cambio es más que dudoso el criterio que ha podido llevar a la inclusión de un Título sobre medios de comunicación social, ya que al tratarse de una materia que se encuadra en el ámbito constitucional de la libertad de información no parece el más adecuado para conformar una "política pública" en sentido estricto, sino en todo caso un espacio para introducir las prioridades de control de la propia Junta sobre el sector.

Por último el Título sobre la reforma prevé dos hipótesis, la de la reforma ordinaria que afectaría a las relaciones con el Estado, donde se exige mayoría cualificada de dos tercios del Parlamento andaluz; y la del procedimiento simplificado que se supone no afecta a las relaciones con el Estado donde bastaría aprobación (no se establece con qué tipo de mayoría) del Parlamento andaluz y posterior "consulta" a las Cortes. Aunque en ambos casos se exige finalmente referéndum ratificatorio y aprobación como Ley Orgánica.

\section{Consideraciones finales}

Las exigencias de reforma del sistema territorial español se situaban a finales del siglo XX no tanto en la demanda de un mayor autogobierno sino más bien en la búsqueda de un mejor sistema de coordinación general entre las partes y el todo: un ámbito donde el reiterado fracaso del Senado, la insuficiencia de las Conferencias Sectoriales y la continuada dinámica conflictual o anti-centro, unida a las inercias centralistas de la administración central, daban lugar a un panorama caracterizado por sus sonadas insuficiencias, apenas amortiguadas por una jurisprudencia constitucional a veces sobrecargada y generalmente muy retrasada en el tiempo. 
Sin embargo la salida a esta situación problemática no parece haber consistido en tratar de mejorar "el sistema" sino más bien avanzar en la reforma de cada una de sus partes, hacia el horizonte de un mayor autogobierno. Lo que suscita de inmediato la perspectiva de que las posibilidades de coordinación general del sistema territorial seguirán siendo progresivamente más problemáticas en el futuro; en particular teniendo en cuenta la peligrosa deriva hacia instrumentos de relación bilateral que preludia la emergencia de un difuso modelo confederal claramente alejado de las previsiones constitucionales.

Naturalmente todo proceso de reforma tiene otra cara, en principio más amable, que consiste en la pura actualización modernizadora de sus contenidos, operando desde la perspectiva interna; ya sea mediante la incorporación de elementos desarrollados con anterioridad por vía legal, ya sea mediante la introducción de nuevos argumentos retóricos o discursivos, más adecuados al contexto sociocultural de principios del siglo XXI. En este caso, la reforma afectaría pues más bien a la dimensión cosmética de la normativa fundamental y no tanto a sus contenidos sustanciales.

Por otra parte la pretensión de caminar por la "vía catalana" del agotamiento competencial provoca un resultado seguramente no previsto: la inevitable expansión de las pautas de presencia pública en numerosos ámbitos materiales (con el objetivo inicial -se supone- de evitar interferencias del estado central), dando así la apariencia de un inexorable incremento del intervencionismo público. En el caso andaluz, y si recordamos el protagonismo que durante la fase inicial de elaboración tuvieron el PSOE e Izquierda Unida, puede tratarse incluso de un objetivo deliberado, pues esa expansión de los ámbitos de actuación pública se refleja en la abundancia de algunos de los recursos estilísticos al uso en este ámbito (fundamentalmente el manejo del futuro verbal como expresión de mandatos inexorables: los poderes públicos "impulsarán", "protegerán" "fomentarán", etc.) unidos a la deliberada apuesta por ubicar los ámbitos prestacionales bajo la cobertura garantista de servicios públicos (generalmente "de calidad"). Todo lo cual significa, a la postre, que el nuevo Estatuto apenas reconoce espacios de autonomía o protagonismo activo al "tercer sector", integrando tanto al voluntariado como en general a la sociedad civil organizada. Lo que no deja de suscitar una cierta contradicción, cuando algunos de los nuevos valores prioritarios que se pretenden consolidar parecen ser la participación o el estímulo de la capacidad emprendedora.

Se trata de una apuesta cuya congruencia histórica no deja de suscitar algunas interrogantes si tenemos en cuenta que la evolución del futuro del estado del bienestar tiene algunos de sus desafíos abiertos más significativos en este ámbito, en el papel de la sociedad civil y del tercer sector, dando por superada la fase histórica de apogeo de "lo público" y del modelo de servicios públicos universales, más característica del siglo XX. Por otra parte habría que considerar la reflexión general de que una normativa fundamental no debería "cerrar" las opciones en cuanto a posibles modelos de acción pública para atender al nuevo sistema de objetivos programáticos o valores y derechos sociales regulados, pues estos deberán depender del distinto tipo de mayorías existentes en cada momento.

Esta apuesta deliberada por la reserva al sector público de la mayor parte de los ámbitos competenciales asumidos se complementa finalmente con la presencia dominante de contenidos programático-finalistas que, sin embargo no aparecen suficientemente compensados por inno- 
vaciones en los soportes o elementos instrumentales con los que deberá hacérseles frente. Y es que, en general, en la evolución histórica del estado de bienestar subyace una cierta polémica acerca de si la línea más eficiente para asegurar su futuro debe consistir en incrementar los fines o más bien en incrementar los medios o instrumentos de acción. La apuesta del nuevo Estatuto de Autonomía de Andalucía parece preocupada en forma bastante miope por incrementar los objetivos, fines o derechos, mientras la interrogante de cuáles serán los "nuevos" instrumentos con los que deberá atenderse a su satisfacción efectiva no parece tener respuesta adecuada. De ahí su reiterada dimensión programática que atraviesa prácticamente todo el articulado.

En definitiva cabe afirmar que la creación de nuevos espacios públicos por parte del Estatuto de 2007 no resulta ser al final ni suficientemente innovadora ni adecuadamente equilibrada en su diseño, al menos en la medida en que la sociedad civil andaluza parece relativamente ausente en las previsiones futuras de la acción pública que deberá enfrentar la Junta de Andalucía.

\section{Bibliografía}

AJA, E. (2003), El estado autonómico. Federalismo y hechos diferenciales, $2^{a}$ ed., Madrid, Alianza.

BlANCO, R. (2004), Nacionalidades históricas y regiones sin historia, Madrid, Alianza.

Cámara, G., Cano, J, (eds.) (1993), Estudios sobre el Estado Social. El Estado social y la Comunidad Autónoma de Andalucía, Madrid, Tecnos, Parlamento de Andalucía.

Ferraro, F. (coord.) (2006), "Un balance del estado de las autonomías", Mediterráneo Económico, $\mathrm{n}^{\circ} 10$.

INSTITUT d'Estudis AutONOMics (2003), Informe sobre la reforma del Estatuto, Barcelona, Generalitat de Cataluña.

Parlamento de Andalucía (2003), XX Aniversario del Estatuto, Granada, Comares (Varios volúmenes).

Pérez Yruela, M. (2002), "Para una nueva teoría de Andalucía: cambio y modernización en la sociedad andaluza", en E. Moyano Estrada, M. Pérez Yruela, (eds.) La Sociedad Andaluza [2000], Córdoba, IESA.

Porras, A. (2006), "El debate territorial español a comienzos del siglo XXI", Revista de Fomento Social, $\mathrm{n}^{\circ} 242$.

- (2005), "La reforma de la administración andaluza y los desafíos de la Segunda Modernización, Revista de Fomento Social, n² 237.

- (2003), "Del proceso autonómico hacia un sistema de red. (Regiones y Comunidades Autónomas en los albores del siglo XXI), Revista de Fomento Social, n 229. 
Porras, A., Ruiz-RIco, J. J. (eds.), (1990), El Estatuto de Andalucía. Estudio sistemático, Barcelona, Ariel.

Revista JuRídica de CAstilla y león (2005), "La Reforma de los Estatutos de Autonomía", Valladolid, Junta de Castilla y León.

Revista Valenciana d'Estudis Autonomics (2007), "Un Estatuto para el siglo XXI" (2 vols.), № 49/50, 2005.

Roura, S, Tajadura, J. (dirs), (2005), La reforma constitucional, Madrid, Biblioteca Nueva.

Ruiz Robledo, A. (1991), El ordenamiento jurídico andaluz, Madrid, Cívitas.

SublRats, J., Gallego, R. (eds.), (2002), Veinte años de autonomías en España. Leyes, políticas públicas, instituciones y opinión pública, Madrid, CIS.

Terol Becerra, M. J. (coord.) (2005), El Estado autonómico 'in fieri'. La Reforma de los Estatutos de Autonomía, Sevilla, IAAP.

Vidal, J. M. García Herrera, M. A. (eds.) (2005), El Estado autonómico: integración, solidaridad, diversidad, Madrid, Colex/INAP. 